\title{
Single-Molecule Junction Conductance through Diaminoacenes
}

Jordan R. Quinn, Frank W. Foss Jr., Latha Venkataraman,* Mark S. Hybertsen, and

$$
\text { Ronald Breslow* }
$$

Department of Chemistry, Department of Physics and Department of Applied Physics and Applied Mathematics, and Center for Electron Transport in Molecular Nanostructures, Columbia University, New York, NY 10027, and Center for Functional Nanomaterials, Brookhaven National Laboratory, Upton, NY 11973

\section{Supplementary Information}

\section{Contents: \\ 1. Experimental Procedures \\ 2. Conductance Histograms \\ 3. Theoretical Procedures}




\section{Experimental Procedures}

Reagents and starting materials obtained from commercial sources were typically $\geq 97 \%$ purity. 1,3-Diaminobenzene and 1,4-diaminobenzene were purchased from SigmaAldrich and were used without further purification. 1,4-Diaminonaphthalene and 1,5diaminonaphthalene were purchased from Sigma-Aldrich and were sublimed under vacuum $\left(<200{ }^{\circ} \mathrm{C}\right)$ prior to use. 2,6-Diaminonaphthalene, ${ }^{1}$ 2,7-diaminonaphthalene, ${ }^{2}$ 2,6diaminoanthracene $^{3}$ and 9,10-diaminoanthracene ${ }^{4}$ were synthesized according to published methods and were sublimed under vacuum $\left(<200^{\circ} \mathrm{C}\right)$ prior to use.

\section{Conductance Histograms}

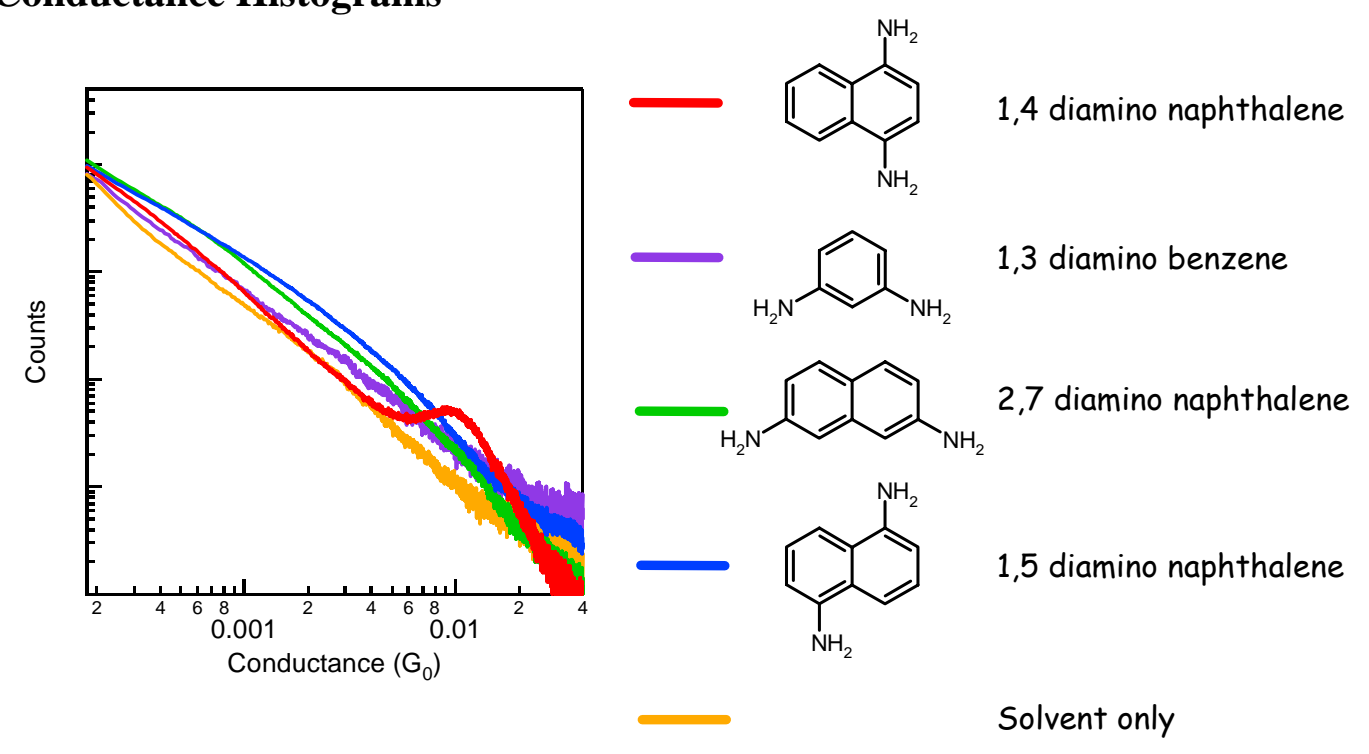

9,10-Diaminoanthracene reacts with molecular oxygen in the air to form the corresponding 9,10-bisimine. ${ }^{4}$ The experimental conductance value for 9,10diaminoanthracene was determined from the average of multiple runs that generally produced several hundred conductance traces. The higher reactivity of 9,10diaminoanthracene with oxygen is reflected in the larger error of the conductance value.

\section{Theoretical Procedures}

The generalized gradient approximation (GGA) as formulated by Perdew, Burke and Ernzerhof (PBE) was used. ${ }^{5}$ The molecular calculations were done with Jaguar v5.0 using a $6-31 \mathrm{~g} * *$ basis for the light atoms and a lacvp** basis for Au. ${ }^{7}$ The molecular geometry was fully relaxed. Each amine-Au link was modeled using a single Au atom. For 1,4-diaminobenzene, Au binds to the amines in a trans configuration. All of the molecules were studied in this configuration.

For the sequence 1,4 diaminobenzene, 1,4 diaminonaphthalene, 9,10 diaminoanthracene, 5,12 diaminotetracene and 6,13 diaminopentacene, the amine-Au link is characterized by a N-Au bond length of 2.43-2.42 $\AA$ and a Au-N-C bond angle of 121-120 degrees. The energy cost to remove the two $\mathrm{Au}$ atoms from the complex systematically rises across 
this sequence by $0.2 \mathrm{eV}$. This correlates to a systematic drop in the calculated ionization potential from $6.5 \mathrm{eV}$ to $5.3 \mathrm{eV}$ for the diamino species.

For the sequence 2,6 diaminonaphthalene, 2,6 diaminoanthracene, 2,8 diaminotetracene, and 2,9 diaminopentacene, the amine-Au link is characterized by a N-Au bond length of 2.37-2.40 $\AA$ and a Au-N-C bond angle of 107 - 111 degrees. The energy cost to remove the two Au atoms from the complex systematically drops across this sequence by $0.4 \mathrm{eV}$. This correlates to a systematic drop in the calculated ionization potential from $6.2 \mathrm{eV}$ to $5.3 \mathrm{eV}$ for the diamino species and a corresponding increase in delocalization of the $\mathrm{N}$ lone pair density into the aromatic system.

The formula in the text is valid for a Huckel model with constant electronic coupling $V$ between $\pi$ electrons on neighboring $\mathrm{C}$ sites, neglecting the overlap. The transmission is derived by an iterative procedure, based on the expression for the bridge molecule Green's function, ${ }^{8}$ including the self energy representing electronic coupling to the electrodes $(-i \Gamma / 2)$ at the 2 and 6 positions. The formula represents the linear response conductance, assuming that the electrode Fermi energy is aligned to the middle of the bridge molecule HOMO-LUMO gap. For reference, the same model yields the following formula for the conductance transverse to the acene axis, through the center ring, for an odd number of rings.

$$
\mathrm{G}=\mathrm{G}_{0}(\mathrm{r}+1)^{2} \Gamma^{2} \mathrm{~V}^{2} /\left(\mathrm{V}^{2}+(\mathrm{r}+1)^{2} \Gamma^{2} / 16\right)^{2}
$$

Because $\Gamma<<V$, this represents the rise in conductance with acene length for the transverse case. More generally, the dependence on length does depend in detail on the final energy alignment of the electrode Fermi energy relative to the center of the HOMOLUMO gap as well as the intervening amine group.

\footnotetext{
${ }^{1}$ Chatt, J.; Wynne, W. P. J. Chem. Soc. 1943, 33-36.

${ }^{2}$ Bell, F.; Gibson, J. A.; Wilson, R. D. J. Chem. Soc. 1956, 2335-2340.

${ }^{3}$ Rabjohns, M. A.; Hodge, P.; Lovell, P. A. Polymer 1997, 38, 3395-3407.

${ }^{4}$ Schiedt, v. B. J. Prakt. Chem. 1941, 157, 203-224. Campbell, T. W.; Mccoy, V. E.; Kauer, J. C.; Foldi, V. S. J. Org. Chem. 1961, 26, 1422-1426.

${ }^{5}$ Perdew, J. P.; Burke, K.; Ernzerhof, M. Phys. Rev. Lett. 1996, 77, 3865-3868.

${ }^{6}$ Jaguar Jaguar 5.0; Schrödinger, L.L.C., Portland, OR, 1991-2003.

${ }^{7}$ Wadt, W. R.; Hay, P. J. J. Chem. Phys. 1985, 82, 284-298.

${ }^{8}$ Nitzan, A. Ann. Rev. Phys. Chem. 2001, 52, 681-750.
} 